\title{
USO OFF LABEL DO BEVACIZUMAB
}

USE OFF LABEL OF BEVACIZUMAB

\author{
BIAZI, Victória ${ }^{1}$; VALDIVINO, Ivana ${ }^{1}$; MENESES, Eli Cristiano \\ ${ }^{1}$ Graduadas em Farmácia pela Universidade São Francisco. \\ ${ }^{2}$ Professor do Curso Farmácia - Universidade São Francisco.
}

\section{victoriatosto58@gmail.com}

\begin{abstract}
RESUMO. Com a progressão demográfica da população e o aumento da expectativa de vida, doenças que não eram tão prevalentes se tornaram mais comuns, como é o caso da doença macular relacionada à idade. Há duas formas: a não-exsudativa e a exsudativa, sendo esta a mais grave, devido ao crescimento anormal de novos vasos no espaço sub-retiniano ocorrendo o extravasamento de sangue, lipídios e plasma, levando assim, a perda de visão. A DMRI é, atualmente, a principal causa de cegueira nos países industrializados, mas em âmbito global fica atrás apenas de catarata e glaucoma. Uma alternativa e que tem se tornado terapia de primeira-linha é o uso off label do Bevacizumabe. O uso off label tem ganhado espaço nos dias de hoje devido a farmacoeconomia, que visa avaliar o custo-benefício dos medicamentos em relação ao valor e ação terapêutica. Este trabalho trata-se de uma revisão bibliográfica de artigos nacionais e internacionais com o intuito de avaliar e comprovar o custo-benefício do Bevacizumabe em relação aos outros medicamentos para a DMRI.
\end{abstract}

Palavras-chave: Off label; Bevacizumabe; DMRI; Farmacoeconomia

\begin{abstract}
With demographic progression of the population and increased life expectancy, diseases that were not so prevalent have become more common, such as age-related macular disease. There are two forms: the non-exudative and exudative, being this the most serious, due the abnormal growth of new vessels in the sub retinal space, with the leakage of blood, lipids and plasma, thus leading to loss the vision. AMD is currently the main cause of blindness in industrialized countries, but globally it is behind only to cataract and glaucoma. An alternative that has become first-line therapy is the off-label use of Bevacizumab. The off-label use has gained space nowadays due the pharmacoeconomics, which aims to evaluate the cost-benefit of drugs in relation to the value and therapeutic action. This article is a literature review of national and international articles to evaluate and prove the cost-benefit of Bevacizumab in relation to other drugs for AMD.
\end{abstract}

Keywords: Off label; Bevacizumab; AMD; pharmacoeconomics.

\section{INTRODUÇÃO}

A Agência Nacional de Vigilância Sanitária (ANVISA) tem como função principal a proteção da saúde da população por intermédio de atividades de regulação, normatização, controle e fiscalização (ANVISA, 1999).

O termo medicamento off label significa que o mesmo está sendo utilizado para finalidades diferentes das que constam na bula, ou seja, utilizado para indicações, via, frequência de administração, posologia e idade que não foram autorizadas por um agência reguladora, porém, isto não significa que seu uso seja incorreto, apenas não houve estudos clínicos suficientes que comprovem sua indicação até o momento (ANVISA, 2005). 
A degeneração macular relacionada à idade (DMRI) é, em âmbito global, a terceira causa de cegueira ficando apenas atrás de catarata e glaucoma. Porém é a principal causa de cegueira nos países industrializados (WHO).

Pesquisas relataram a prevalência da DMRI em diferentes faixas etárias, sendo 1,2\% dos pacientes com menos de 65 anos, $7 \%$ de 65 a 74 anos de idade e $20 \%$ em pacientes acima de 75 anos (SERRA et al., 2014) Conclui-se que o principal fator desta doença é o aumento da idade. Porém há outros fatores envolvidos como etnia caucasiana, tabagismo e polimorfismos genéticos. Contudo, estudos demonstram que a idade é o fator primordial para o avanço da doença (FIGUEIREDO; FIREMAN, 2018)

Há duas formas de DMRI: seca ou não-exsudativa (90\% dos casos) e úmida ou exsudativa (10\% dos casos) (FIGUEIREDO; FIREMAN, 2018). A forma exsudativa é a mais grave, devido ao desenvolvimento anormal de novos vasos (angiogênese) no espaço subretiniano, havendo a formação de uma membrana denominada membrana neovascular subretiniana (MNSR) (GASS, 1974).

Com o avanço da idade, o epitélio pigmentar da retina (EPR) está com sua capacidade diminuída, assim havendo o acúmulo de metabólitos no espaço sub-retiniano, e também haverá um acúmulo de lipídeos na membrana de Bruch, tornando-se espessa, consequentemente diminuindo sua permeabilidade que causará hipóxia (diminuição de oxigênio). A falta de oxigênio faz com que haja um aumento na produção do fator de crescimento do endotélio vascular ("vascular endothelial growth factor", VEGF), assim estes novos vasos penetraram no espaço sub-retiniano causando exsudação sub e intra retiniana de sangue, lipídios e plasma levando a perda de visão central (DAMICO, 2007).

De acordo com a pesquisa realizada pela American Academy of Ophthalmology nos Estados Unidos, em 2004 aproximadamente 1.75 milhões de pessoas com 40 anos ou mais, tinham DMRI ou atrofia geográfica em pelo menos um olho, e 7.3 milhões eram considerados como grupo de alto risco de ter grandes drusas em ambos os olhos. Outros resultados indicam que as 1.75 milhões de pessoas afetadas pela DMRI avançada em pelo menos um olho, irão aumentar para três milhões até 2020 devido à progressão demográfica da idade da população dos EUA (EMPTAGE et al., 2015).

Atualmente, existem no mercado três medicamentos - inibidores do VEGF - para o tratamento da doença macular relacionada à idade: Ranibizumabe (Lucentis ${ }^{\circledR}$ ), Pegaptanibe e Bevacizumabe (Avastin ${ }^{\circledR}$ ). Sendo, os dois primeiros registrados no Food and Drugs Administration (FDA) e ANVISA para o tratamento de escolha para DMRI, enquanto o Avastin ${ }^{\circledR}$ é indicado para câncer de colo retal metastático, porém vem sendo utilizado como tratamento off label para DMRI, devido atuar inibindo o VEGF (BRATS, 2008).

Os inibidores do VEGF têm demonstrado uma melhora nos resultados visuais e anatômicos quando comparados há outros protocolos terapêuticos. Por conta disto, os antiVEGF se tornaram o tratamento de primeira linha para a DMRI neovascular (KLEIN et al., 2007)

O bevacizumabe, que é o foco do trabalho, é um anticorpo monoclonal anti-VEGF humanizado, que bloqueia a ação do fator de crescimento vascular endotelial, ou seja, impede que haja o crescimento de novos vasos que suprem os tumores malignos, como sua indicação terapêutica para câncer de colorretal metastático. Na DMRI seu uso off label também tem como finalidade o bloqueio do crescimento dos novos vasos, entretanto com outro objetivo: permite que a neovascularização não ocorra, não havendo assim vazamento de líquidos ou sangue, consequentemente melhorando a visão ou apenas estabilizando o caso. (AVASTIN, 2005) 
A Farmacoeconomia (FE) é uma área que aplica a economia no estudo dos medicamentos/terapias medicamentosas, visando o custo-benefício, ou seja, a otimização dos recursos financeiros e a qualidade do tratamento. Para se definir qual medicamento será escolhido dependerá dos benefícios, o custo do tratamento e os riscos que levarão para os pacientes. Além disso, pode-se dizer que a FE leva em consideração a segurança, eficácia e qualidade de medicamentos juntamente com os custos econômicos, sendo uma ferramenta indispensável para a distribuição mais equilibrada dos recursos sanitários de um país (ZANINI et al., 2001).

$\mathrm{Na}$ área da oncologia, por ter uma variedade extensa e uma entrada de novos medicamentos com um custo cada vez mais elevado, a aplicação da farmacoeconomia é essencial e interessante. Na saúde complementar este sistema é utilizado, porém com menor ênfase que na saúde pública, devido este depender de verbas governamentais e não somente do benefício terapêutico, mas sim da análise de custos e das vantagens que tal medicamento trará para o indivíduo (POMBO, 2015).

Por conta destes fatores, o objetivo do presente trabalho é evidenciar que o uso off label do medicamento bevacizumabe, conhecido comercialmente por Avastin ${ }^{\circ}$, que é equivalente ao ranibizumabe (Lucentis $\left.{ }^{\circledR}\right)$ em relação a sua eficácia na doença macular relacionada a idade (DMRI). Além de comparar os valores monetários dos tratamentos com as duas medicações.

\section{METODOLOGIA}

O trabalho foi realizado através de um levantamento de dados bibliográficos, pesquisa com bases de incidência epidemiológicas, revisão de artigos acadêmicos e literários indexados na Scielo Scientific Eletronic Library Online, Pubmed, Google acadêmico e nos manuais da Sociedade Brasileira de Farmacêuticos em Oncologia (SOBRAFO), além das agências reguladoras: Agência Nacional de Vigilância Sanitária (ANVISA) e Food and Drugs Administration (FDA) e monografia do produto Avastin ${ }^{\circledR}$ do laboratório Roche.

Foram utilizados como palavras-chave de busca destes artigos: bevacizumabe, off label, doença macular relacionada à idade (DMRI) farmacoeconomia e VEGF.

O período de busca destas referências bibliográficas para a composição deste trabalho foi compreendido entre 1974 e 2015.

Os critérios de inclusão dos trabalhos pesquisados para serem utilizados nesta pesquisa foram os estudos que evidenciaram o uso off label do bevacizumabe na DMRI.

\section{RESULTADOS E DISCUSSÃO}

O uso off label como já foi citado, é o uso do medicamento para uma indicação, faixa etária, dose ou via de administração diferente do que foi aprovado pelos órgãos reguladores, no caso ANVISA no Brasil e FDA nos EUA (GAZARIAN et al., 2006).

Um estudo analisou o banco de dados do National Disease and Therapeutic Index (NDTI), que reportou uma estimativa de 725 milhões de menções totais de medicamentos incluídos na amostra em 2001, e deste total $21 \%$ não tinha a aprovação do FDA, ou seja, 150 milhões de menções destes medicamentos foram off label (RADLEY; FINKELSTEIN; STAFFORD, 2006).

Alguns exemplos podem ser citados, como: o albuterol que é antiasmático, mas é utilizado como indicação off label para tose crônica; o propanolol que tem indicação terapêutica de anti-hipertensivo e antiarrítmico porém é usado como off label para ansiedade; outro 
exemplo bem difundido na sociedade são os fármacos da família do sidenafil/vardenafil/tadalafil que, na bula, sua indicação é para tratamento de disfunção erétil mas seu uso off label e o mais comum é para aumentar a performance sexual em indivíduos sem disfunção erétil (VAZ CARNEIRO; COSTA, 2013).

O tratamento para a DMRI exsudativa se divide em duas frentes: o não medicamentoso, que se baseia em terapias destrutivas como: fotocoagulação a laser e terapia fotodinâmica; e o medicamentoso que possui algumas alternativas no mercado, como: Pegaptanibe sódico, Ranibizumabe e Aflibercepte, que são tratamentos aprovados pela ANVISA e FDA para a DMRI exsudativa. Há também o Bevacizumabe, que é de uso off label para a mesma doença porém não é aprovado pelos órgãos regulatórios para a DMRI (FIGUEIREDO; FIREMAN, 2018).

O uso off label do bevacizumabe para esta doença, começou a ser estudado em 2005 por Rosenfeld e colaboradores, em um estudo chamado SANA (Systemic Avastin for Neovascular AMD). Este primeiro trabalho tinha como método a infusão intravenosa (IV) do Avastin $(5 \mathrm{mg} / \mathrm{kg})$ seguida de uma ou duas doses adicionais administradas em um intervalo de duas semanas, porém após o término dos testes foi encontrado prós e contras sobre o bevacizumabe intravenoso para a DMRI. Os resultados obtidos a partir do SANA mostraram que o Avastin IV tem uma eficácia similar ou melhor que o ranibizumabe intraocular após duas ou três aplicações e os benefícios adquiridos permaneceram por seis meses ou mais sem necessidade de outras aplicações (MICHELS et al., 2005).

A desvantagem dessa via de administração para este caso é o valor, pois como é intravenosa a quantidade de miligramas necessária para ocorrer o efeito desejado é alto, tendo um custo aproximado de $\$ 2220$ por infusão. Outro fator que leva a ser desvantajoso é a probabilidade de, nesta via de administração, ocorrer hipertensão, eventos tromboembólicos, ataque cardíaco ou até mesmo derrame. Mesmo não tendo sido comprovados com números essas probabilidades, só o receio de ocorrer, fez com que essa alternativa de Avastin IV com doses altas, mudasse a opinião dos pesquisadores, mesmo com resultados satisfatórios. Assim tiveram que pensar em outra via de administração do medicamento para assim, diminuir tais riscos e custos, o que levou a escolha, atualmente da injeção intravítrea (ROSENFELD, 2006).

A idéia da injeção intravítrea do Avastin surgiu, como o próprio Rosenfeld disse em uma entrevista, como um momento "eureka". Ele estava dirigindo para casa pensando em como o Avastin poderia ser entregue em segurança no olho, sem muitos efeitos colaterais, e foi ai que o momento "eureka" aconteceu, quando ele pensou que a quantidade necessária de Avastin poderia ser injetada no olho seguindo o mesmo volume baixo do Lucentis, porém com custo menor. Assim, no dia posterior, Rosenfeld foi conversar com o diretor farmacêutico da universidade de Miami, onde os testes estavam sendo realizados, para preparar as injeções (STEINBROOK, 2006).

Esta mudança de via de administração, após a idéia de Rosenfeld, que ocorreu em meados de maio de 2005, fez com que iniciasse um novo teste para avaliar a capacidade terapêutica do bevacizumabe em injeção intravítrea. Em julho do mesmo ano, Rosenfeld e colaboradores, publicaram um estudo mostrando as vantagens e benefícios dessa aplicação após sucesso nos testes em dois pacientes. Após esta publicação, em menos de seis meses, o uso do Avastin para este fim, se difundiu pelo mundo (STEINBROOK, 2006).

A grande aceitação do bevacizumabe intravítreo se deve pelo fato da semelhança entre esta molécula e a molécula do ranibizumabe, sendo as duas derivadas do mesmo anticorpo monoclonal anti-VEGF do rato, porém o ranibizumabe é apenas uma fração do anticorpo, já o bevacizumabe é o completo (STEINBROOK, 2006). 

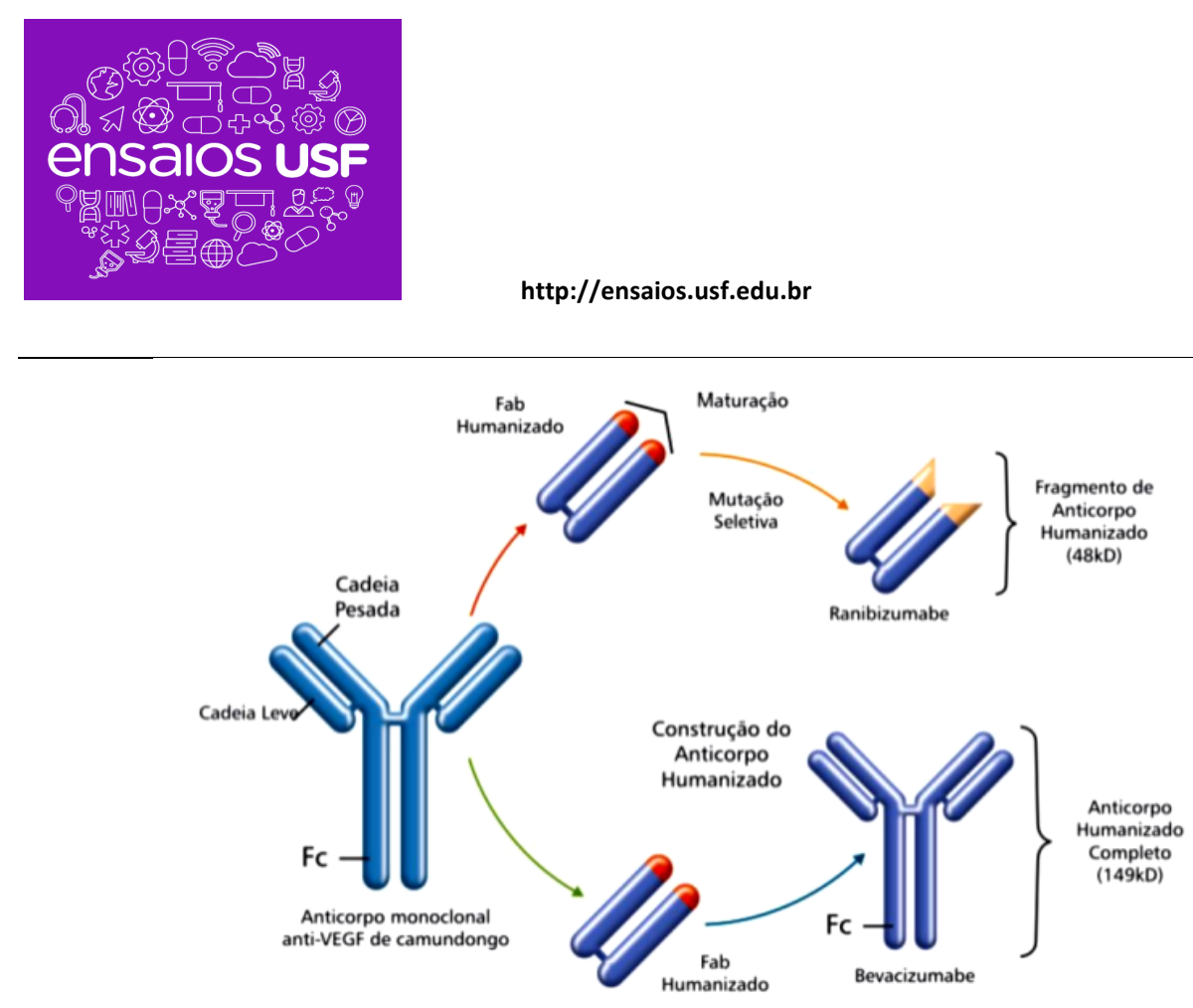

Figura 1- Relação entre ranibizumabe e bevacizumabe (Fonte: adaptado de STEINBROOK, 2006).

O bevacizumabe é um anticorpo monoclonal Ig1 humanizado que é produzido no organismo de um camundongo chinês (molécula glicosilada), tendo peso molecular de $149 \mathrm{kD}$ pois possui além da porção $\mathrm{Fab}$, a porção constante $\mathrm{Fc}$; já o ranibizumabe é um fragmento (Fab) do mesmo anticorpo produzido no sistema de expressão da Escherichia coli, tendo peso molecular de $48 \mathrm{kD}$ (molécula não glicosilada), sendo produzido especialmente para uso intravítrea (STEINBROOK, 2006).

Os dois tipos de medicamentos, seja ele o anticorpo completo ou apenas a porção Fab, inibem todas as formas do VEGF, porém o ranibizumabe tem maior afinidade na ligação com fator de crescimento e consequentemente, maior inibição do mesmo. Isto é devido à mutação seletiva feita em laboratório para fazer com que este medicamento atue apenas inibindo este fator, assim diminuindo os possíveis efeitos colaterais (STEINBROOK, 2006).

A diferença de peso molecular entre os medicamentos faz com que haja algumas mudanças na farmacocinética. Uma das mudanças é a meia-vida sistêmica, onde o ranibizumabe tem uma meia-vida de dois dias e o bevacizumabe de vinte dias. Outra mudança é que após a aplicação intravítrea do bevacizumabe é possível verificar na corrente sanguínea, fragmentos farmacologicamente ativos, já do ranibizumabe essas quantidades são muito baixas ou indetectáveis. Além disto, a meia-vida intravitrea dos dois diferem-se, sendo do ranibizumabe 2,88 horas e do bevacizumabe 4,32 horas (NOVARTIS BIOCIÊNCIAS S.A, 2014).

Para avaliar as diferenças farmacológicas dos dois medicamentos em questão, bevacizumabe e ranibizumabe, foi e é necessário que haja testes para avaliar a semelhança de resultados obtidos a partir de certo tempo de tratamento, para assim aprovar o Avastin ${ }^{\circledR}$ como forma alternativa de tratamento para a DMRI. Essas semelhanças envolvem alguns tópicos a serem considerados, como: ganho de letras, taxa de manutenção, ganho de acuidade visual (AV), redução da espessura foveal, além do número médio de aplicações referente a cada medicamento (GROUP, 2011).

A acuidade visual (AV) é o que define a capacidade do olho enxergar, com detalhes, o contorno e a forma dos objetos. Ela é realizada através de escalas, como a Tabela de Sneellen, que fica posicionada há 6 metros (20 pés) do paciente e é requerido que o mesmo leia os optotipos de diversos tamanhos, para avaliar qual é a AV, sendo 20/20 a visão considerada 
normal, que significa que uma pessoa consegue enxergar detalhes a20 pés iguais uma normal enxerga (SILVA, 2014)

O estudo da equivalência do Avastin ${ }^{\circledR}$ em relação ao Lucentis ${ }^{\circledR}$ é algo relativamente novo no mercado, por conta disto não há uma diversidade de artigos e estudos sobre a semelhança entre eles. Há, entre eles os mais relevantes e os que são utilizados como referências para atestar uso do bevacizumabe em redes públicas e privados, levando em consideração o custo-benefício de cada um.

Os testes que são relevantes para avaliar o custo-benefício do Avastinß ${ }^{\circledR}$ são descritos a partir de 2011, onde foram realizados estudos randomizados, ou seja, os participantes do estudo foram escolhidos de forma aleatória de acordo com os critérios estabelecidos em cada teste. Dentre eles podemos citar quatro: CATT, IVAN, GEFAL e MANTA.

O estudo CATT (Comparison of Age-Related Macular Degeneration Treatment Trials), foi publicado em 2012, sendo o pioneiro no assunto de bevacizumabe versus ranibizumabe, onde foi avaliado a segurança e eficácia do ranibizumabe em relação ao bevacizumabe. No artigo publicado em 2012, que abrangeu dois anos de testes, o objetivo era descrever os efeitos destes medicamentos quando fossem administrados mensalmente e conforme a necessidade por dois anos e o impacto que teria se trocasse, após um ano de tratamento, o que era aplicado mensalmente para conforme a necessidade (MARTIN et al., 2012).

Os critérios de elegibilidade para participar do teste foram: ter 50 anos ou mais; presença de neovascularização coróide ativa no olho de estudo previamente não tratado devido a DMRI; acuidade visual de 20/25 a 20/230. Já os critérios de inclusão eram: possuir neovascularização, fluído ou hemorragia sob a fóvea (GROUP, 2011).

O método desse teste foi realizado da seguinte maneira: os pacientes foram divididos em seis grupos distintos e aleatórios que faziam uso tanto do ranibizumabe e bevacizumabe porem em esquemas de dosagens diferentes, mensal ou conforme a necessidade, para assim avaliar qual seria o melhor método a ser utilizado. Levando em consideração que, o ranibizumabe era uma dose de $0,5 \mathrm{mg}(0,05 \mathrm{~mL})$ e o bevacizumabe era $1,25 \mathrm{mg}(0,05 \mathrm{~mL})$ (MARTIN et al., 2012).

O principal resultado obtido neste teste foi em relação à acuidade visual, onde a maior mudança foi no primeiro ano de tratamento com um pequeno aumento para o segundo ano. Pode-se constatar tal melhora, com o dado de que 60\% dos pacientes - de todos os grupos tinham melhorado sua visão para no mínimo 20/40 (MARTIN et al., 2012).

As duas drogas reduziram de modo substancial e imediato o líquido na retina ou sob ela, porém ao se utilizar o ranibizumabe por um ano, houve uma resolução completa do líquido em mais olhos do que o bevacizumabe. Passados dois anos de testes, as diferenças encontradas em relação à espessura média da retina e a proporção de pacientes com líquido residual continuaram basicamente inalteradas desde o primeiro ano de estudo. Sobre os regimes testados - mensal e conforme a necessidade - quando comparados com o crescimento de neovascularização coroidal, o mensal resultou em um menor crescimento se comparado com o conforme necessidade (MARTIN et al., 2012).

Sobre os efeitos adversos, eles perduraram do primeiro ano para o segundo, seguindo a mesma proporção. As taxas de morte, infarto do miocárdio e acidente vascular cerebral (AVC) não se diferiram se comparadas as duas drogas. A maior taxa de eventos adversos graves relatados no primeiro ano foi do grupo que foi tratado com bevacizumabe, e esta taxa permaneceu no ano seguinte - ano dois de tratamento, mas não diferindo de esquema de dosagem (MARTIN et al., 2012). 
Os testes demonstraram que as taxas desses eventos são baixas, porém há ainda certa preocupação dos autores em relação aos efeitos que o bevacizumabe pode trazer ao paciente, pois ao excluir todos os efeitos relacionados ao VEGF, ainda há indícios de desequilíbrios que podem ser decorrentes de acaso, fatores basais não avaliados ou por causar um risco maior que o ranibizumabe (MARTIN et al., 2012).

Outro fator levado em consideração neste teste foi o valor dos medicamentos, pois, nos EUA o ranibizumabe representou $10 \%$ dos gastos com medicamentos nos planos de saúde. Por conta disto, a escolha do medicamento leva em consideração o custo-benefício (efeitos na visão, possibilidade de verdadeiras diferenças em relação aos efeitos adversos e o custo) - sendo o Lucentis ${ }^{\circledR} 40$ vezes mais caro que o Avastin ${ }^{\circledR}($ MARTIN et al., 2012).

Sobre o estudo, conclui-se que os dois medicamentos tiveram efeitos semelhantes na AV no período de dois anos. Em relação aos esquemas de dosagens, o tratamento conforme a necessidade mostrou um menor ganho na AV em comparação ao mensal. As taxas de morte e eventos arteriotrombóticos ao usar tanto ranibizumabe ou bevacizumabe, não houve diferenças significativas. A falta de especificidade das condições associadas à inibição do VEGF faz com que, a persistência das taxas mais altas de eventos adversos graves relacionados com o bevacizumabe seja algo incerto de ser afirmar (MARTIN et al., 2012).

Outro estudo, chamado IVAN (Inhibit VEGF in Age-related Choroidal Neovascularization) de 2012, tinha como objetivo comparar a eficácia e a segurança do ranibizumabe e bevacizumabe. O método utilizado para teste foi dividir os pacientes em quatro grupos, de acordo com o medicamento escolhido e de acordo com o regime de doses, mensal (contínuo) ou conforme a necessidade (descontínuo) (CHAKRAVARTHY et al., 2012).

Após um ano de testes, a comparação das duas drogas em relação à $A V$, foi inconclusiva, sendo a diferença média entre as drogas, duas letras a favor do ranibizumabe. A diferença na AV comparando os regimes de doses foi insignificante, concluindo assim, a equivalência entre eles. O estudo avaliou também a quantidade de VEGF sérico, assim puderam confirmar que uma droga intravítrea pode sim, passar para a circulação. Em um ano de tratamento, o bevacizumabe teve o índice mais baixo de VEGF sérico - comparado ao ranibizumabe - e isso pode ser explicado por conta dele ser um anticorpo humanizado completo anti-VEGF, assim a ligação aos receptores pode ser maior assim tendo uma menor concentração sérica (CHAKRAVARTHY et al., 2012).

Sobre os eventos adversos, os resultados foram promissores quando tratado da segurança dos medicamentos. Eventos arteriotrombóticos ocorreram em $\pm 2 \%$ dos pacientes, sendo o grupo do ranibizumabe mais freqüente, porém não houve diferenças entre os regimes. Em um ano de tratamento, as taxas de mortalidade e probabilidade de ocorrer um evento adverso sistêmico grave não se diferenciaram quando comparadas as drogas e regimes de doses (CHAKRAVARTHY et al., 2012).

O custo é um fator determinante na hora da escolha de um tratamento, sobre isto, o levantamento dos valores sempre é um tópico a ser abordado nos testes. O ranibizumabe de uso contínuo é o tratamento mais caro em comparação aos outros três grupos. Já a diferença de custos entre bevacizumabe contínuo e descontínuo foi insignificante. Deste modo, foi realizada uma projeção de dados que, se houvesse a troca de ranibizumabe para bevacizumabe poderia se economizar $£ 84,5$ milhões - aproximadamente R $\$ 507$ milhões - com base no total de olhos tratados anualmente na Inglaterra (CHAKRAVARTHY et al., 2012).

Os autores do IVAN o comparam ao estudo CATT, para avaliar a veracidade dos resultados. A diferença média na $\mathrm{AV}$ nos dois testes reforça a equivalência dos dois medicamentos, sendo que no regime contínuo o resultado foi melhor que no descontínuo. Sobre 
a espessura da fóvea os resultados são mais favoráveis para o grupo do ranibizumabe e também para o tratamento contínuo. Em relação à segurança dos medicamentos, após analisar e agrupar os dados dos dois testes, a taxa de mortalidade foi mais baixa com o uso do ranibizumabe e, sobre os eventos arteriotrombóticos o bevacizumabe teve as menores taxas (CHAKRAVARTHY et al., 2012).

Portanto, conclui-se que os dois medicamentos possuem benefícios equivalentes a função visual. Porém o ranibizumabe e o regime de aplicação contínuo conferem melhores resultados morfológicos, mas não diferindo na função visual entre os outros grupos (CHAKRAVARTHY et al., 2012).

Já o GEFAL (Grupo de Estudo Frances sobre Avastin versos Lucentis para a DMRI 2013) que é também um estudo randomizado, foi realizado para avaliar e testar a segurança e eficácia da utilização do Bevacizumabe versus Ranibizumabe no tratamento da DMRI. O teste teve duração de um ano e as aplicações dos dois medicamentos eram feitas no regime de conforme a necessidade (KODJIKIAN et al., 2013).

Os critérios de elegibilidade eram aplicados se os pacientes com mais que 50 anos preenchessem alguns critérios de inclusão: acuidade visual com melhor correção entre 20/32 e 20/320 medida em estudo precoce do tratamento da retinopatia a uma distância de 4 metros; DMRI neovascular subfoveal ativa; e área total de neovascularização coróide menor que 12 áreas de disco óptico (KODJIKIAN et al., 2013).

Os pacientes foram designados aleatoriamente para a injeção de bevacizumabe $(1,25$ $\mathrm{mg} / 0,05 \mathrm{~mL})$ ou ranibizumabe $(0,5 \mathrm{mg} / 0,05 \mathrm{~mL})$. O estudo consistiu em um período de dosecarga (onde se aplicava três injeções nos meses zero, um e dois), e um período de manutenção, referente há nove meses (mês 3 ao 12, começando de 28 a 35 dias após a última injeção de carga). O período de manutenção, onde o paciente receberia ou não outra injeção, dependia se estava presente pelo menos um dos critérios a seguir: perda de cinco letras em relação a última visita; exsudação ativa em TCO; aumento da área de neovascularização coroidal ou persistência de vazamento na angiografia desde a visita anterior; nova ou persistente hemorragia macular sub-retiniana ou intraretiniana (KODJIKIAN et al., 2013).

$\mathrm{O}$ resultado primário obtido foi que o bevacizumabe não era inferior ao ranibizumabe $\mathrm{e}$ que a diferença entre as duas drogas é de apenas 1,89 letras. Já os resultados secundários abrangem a acuidade visual, que foi melhor nos dois grupos, com um aumento durante o período de dose-carga, permanecendo estável durante os nove meses do período de manutenção; O número de injeções, onde a média foi de 6,8 para bevacizumabe e 6,5 para ranibizumabe; Parâmetros de TCO mostraram que ambas as drogas reduziram a espessura da fóvea; Parâmetros angiográficos mostram que $49 \%$ dos pacientes de bevacizumabe e $41 \%$ do ranibizumabe tiveram vazamento de corante no angiograma além de ter uma ligeira diminuição na área média de NVC entre os dois grupos; Sobre os efeitos adversos, ambos os medicamentos apresentaram algum tipo de evento adverso grave, além de arteriotrombose e hipertensão, porém o número de casos foi bem próximo entre as duas drogas (KODJIKIAN et al., 2013).

Conclui-se que o bevacizumabe não é inferior ao ranibizumabe. Ambos os medicamentos levaram a uma rápida e substancial diminuição do liquido sub e intra retiniano, porém na avaliação final, pacientes que usaram ranibizumabe tiveram mais olhos com resolução completa do que os que usaram bevacizumabe. Sobre a segurança, os dois parecem ser similares, não encontrando diferenças entre óbitos, eventos arteriotromboticos ou eventos trombóticos venosos (KODJIKIAN et al., 2013).

Há outro estudo randomizado que foi realizado para atestar a segurança e eficácia do uso do Bevacizumabe e Ranibizumabe utilizado conforme necessidade no tratamento da DMRI, 
chamado MANTA (Multicentre Anti-VEGF Trial in Áustria - 2013), que teve duração de um ano (EHLERS, 2013).

O estudo foi realizado com 321 pacientes, todos sem prévio tratamento. O protocolo de tratamento escolhido foi o PRN (pro re nata - que significa conforme necessário ou quando a situação surgir) que inclui três meses consecutivos de injeções mensais no início (fase de carreamento) e após, os pacientes só eram tratados (conforme necessidade) se apresentasse alguns sinais como: perda de cinco letras ou mais associada com neovascularização coróide subfoveal (CNV) ativa; nova hemorragia macular; aumento da espessura central da retina na tomografia de coerência ótica (TCO) de $100 \mathrm{~mm}$ ou mais ou líquido persistente na TCO (KREBS et al., 2013).

Após um ano de tratamento, o primeiro resultado observado foi a mudança na acuidade visual melhor corrigida (AVMC) com melhora de sete letras, resultado esse, melhor do que nos dois primeiros testes já realizados: CATT e IVAN (EHLERS, 2013).

Em relação à $\mathrm{AV}$, os resultados após os três meses consecutivos de injeções indicaram um pico nos resultados mostrando um ganho de aproximadamente 6,5 letras para o Bevacizumabe e 6 letras para o Ranibizumabe. Após oito meses, mesmo que insignificante, a mudança média na AV começa a diminuir, sendo 4.9 letras para Bevacizumabe e 4,1 letras para o Ranibizumabe, indicando o uso do tratamento PRN. MANTA é o primeiro estudo, mesmo que com resultados não muito significantes, que mostra que o Bevacizumabe é melhor quando comparado ao Ranibizumabe (EHLERS, 2013).

Também foi possível identificar que os níveis séricos de VEGF nas aplicações realizadas mensais, foram mais baixos com o Bevacizumabe do que com o Ranibizumabe (EHLERS, 2013).

As diferenças encontradas sobre a espessura da retina entre as duas drogas não é significante, pois são basicamente equivalentes. Os resultados encontrados sobre a diminuição da espessura da retina foi equivalente aos encontrados nos estudos CATT e IVAN, confirmando assim a veracidade dos mesmos (KREBS et al., 2013).

Em relação aos efeitos adversos sistêmicos apresentados no estudo MANTA, como por exemplo, eventos arteriotrombóticos, infarto do miocárdio e acidente vascular, o grupo do bevacizumabe possui um número ligeiramente maior de eventos adversos, porém esta diferença não é estaticamente significativa entre as duas drogas (KREBS et al., 2013).

Conclui-se que os resultados encontrados, mesmo com diferenças entre eles, não são considerados significantes em relação ao todo, assim pode-se afirmar neste estudo que o bevacizumabe e o ranibizumabe são equivalentes quando comparado a AVMC, espessura da retina, tamanho da lesão e probabilidade de eventos adversos (KREBS et al., 2013).

A farmacoeconomia é um tema recente no Brasil, mas sua aplicação esta sendo de grande influência no sistema público, pois auxilia na escolha de uma terapêutica medicamentosa e na redução de gastos. Em resumo, sua utilização é de suma importância por avaliar os custos, eficácia, utilidade e a eficiência de tratamentos para a mesma doença (PACKEISER; RESTA, 2014).

A DMRI como mostrada anteriormente, é uma doença que possui diversos tratamentos que auxiliam a sua melhora, sendo, porém, alguns com custos bem elevados e que, uma grande porcentagem de pacientes ou o sistema público não possuem verba suficiente para tratá-la. Neste momento é onde a farmacoeconomia se encaixa, escolhendo um tratamento que é mais acessível e que possua equivalência de resultados em eficácia, segurança e qualidade (ZANINI et al., 2001). 
O BRATS (Boletim Brasileiro de Avaliação de Tecnologias de Saúde), em 2008, levantou os valores de mercado dos medicamentos para esta doença: Pegaptanibe, Ranibizumabe e Bevacizumabe, para assim poder comparar os valores com a efetividade dos medicamentos e assim chegar a uma conclusão sobre qual deles tem o melhor custo-benefício.

$O$ valor total do tratamento de cada medicação inclui:

- Posologia de cada medicação - quantidade, em MG, e número de aplicações;

- Tempo de tratamento;

- Valor do Frasco-Ampola.

De acordo com a Câmara de Regulação do Mercado de Medicamentos - CMED, publicado em $1^{\circ}$ de maio de 2020, o valor do Bevacizumabe com o ICMS do estado de São Paulo (18\%) é de R $\$ 1704,60$ para o frasco-ampola de $25 \mathrm{mg} / \mathrm{mL}$ SOL DIL INFUS IV CT FA VD TRANS X $4 \mathrm{~mL}$, e de R $\$ 6.600,00$ para o $25 \mathrm{mg} / \mathrm{mL}$ SOL DIL INFUS IV CT FA VD TRANS X $16 \mathrm{~mL}$. O Ranibizumabe (Lucentis) de $10 \mathrm{mg} / \mathrm{mL}$ SOL INJ CT 1 FA VD INC X $0,23 \mathrm{~mL}+\mathrm{SER}+\mathrm{AGU}+$ FILTRO P/ INJ, com o ICMS de 18\% do Estado de São Paulo custa $\mathrm{R} \$ 3.994,76$ (preço de fábrica). (ANVISA, 2020).

O valor do Pegaptanibe é referente a 2008, onde $0,3 \mathrm{mg}$ SOL INT-OC CT ENV PLAS X SR VD (PRE-ENCHIDA C/AG) + ENV PLAS X TRAVA PLAS + EMB PLAS possui o preço fábrica de R $\$ 2.260,42$ (BRATS, 2008)

Utilizando os parâmetros do BRATS-2008, para avaliar o valor total do tratamento apresentado no Quadro 1 sobre os preços dos tratamentos:

Quadro 1 - Valores de tratamentos para DMRI

\begin{tabular}{|l|c|c|c|}
\hline \multicolumn{1}{|c|}{ Medicamento } & Posologia & $\begin{array}{c}\text { Tempo de } \\
\text { Tratamento }\end{array}$ & Custo do tratamento \\
\hline Ranibizumabe & $\begin{array}{c}\text { 1 injeção de } 0,5 \mathrm{mg} \text { a cada } \\
4 \text { semanas }\end{array}$ & Três meses & $\mathrm{R} \$ 11.984,28$ \\
\hline Bevacizumabe & $\begin{array}{c}1 \text { injeção de } 1,25 \mathrm{mg} \text { a } \\
\text { cada } 4 \text { semanas }\end{array}$ & Três meses & $\mathrm{R} \$ 5.113,80$ \\
\hline Pegaptanibe & $\begin{array}{c}1 \text { injeção de } 0,3 \mathrm{mg} \text { a cada } \\
6 \text { semanas }\end{array}$ & Doze meses & $\mathrm{R} \$ 20.343,78$ \\
\hline
\end{tabular}

Fonte: Próprio autor

O valor final do tratamento levou em consideração o uso de um frasco por aplicação. Mas, segundo o documento do BRATS - 2008, o bevacizumabe é hoje em dia fracionado em doses unitárias, assim vendido para as clínicas oftalmológicas em seringas prontas com a dose que será aplicada no paciente $(1,25 \mathrm{mg}-0,05 \mathrm{~mL})$, podendo assim diminuir ainda mais o valor de cada dose de bevacizumabe (BRATS, 2008).

O documento do BRATS-2008 afirma que a unitarização das doses do bevacizumabe é possível se seguidas normas técnicas que foram aprovadas a partir de estudos de estabilidade. De acordo com os estudos realizados por Chen et al. (2009), avaliou-se a estabilidade, esterilidade e a eficácia de comercializar o bevacizumabe em seringas armazenadas por até seis meses em temperatura de $4^{\circ} \mathrm{C}$. Os resultados obtidos foram que em todas as amostras de $1,3 \mathrm{e}$ 6 meses não houveram crescimento microbiológico, ficaram estáveis e não houveram mudanças no peso molecular. Conclui-se que, em armazenamento a $4^{\circ} \mathrm{C}$ e preparado em condições assépticas adequadas, o bevacizumabe é estéril e estável (CHEN et al., 2009). 
As condições de preparo da droga devem seguir a RDC n ${ }^{\circ} 220$ de 2004 que dispõe das normas técnicas para se manipular medicamentos antineoplásicos, e a RDC nº 67 de 2007 , que dispõe sobre as boas práticas de manipulação de preparações magistrais e oficinais.

A unitarização das doses faz com que o preço diminua e consequentemente o valor do tratamento reduza. Levando em consideração a farmacoeconomia, o Bevacizumabe é o fármaco que tem maior custo-benefício dos três citados, tendo melhor valor de mercado (e podendo ser fracionado - um FA de $4 \mathrm{~mL}$ rende aproximadamente 80 doses) e sua eficácia e segurança são equivalentes ao Ranibizumabe, como demonstrado em estudos (FIGUEIREDO; FIREMAN, 2018).

\section{CONCLUSÃO}

A doença macular relacionada à idade atinge uma grande parcela da população, sendo a principal causa de cegueira nos países industrializados. Com a expectativa de vida aumentando, o número de casos tende a aumentar e com isso haverá um impacto orçamentário, por conta dos altos valores dos tratamentos disponíveis. Com o estudo da farmacoeconomia, consegue-se avaliar o melhor tratamento com o melhor custo, sem prejudicar a saúde do paciente, o chamado custo-benefício. O uso off label do Avastin ${ }^{\circledR}$ se enquadra como o tratamento com o melhor custo-benefício comprado com as outras terapias já conhecidas para a DMRI, devido ao seu valor de mercado e com a possibilidade de fracionar as doses. Em relação à eficácia e segurança, o bevacizumabe é equivalente, em vários testes realizados, ao ranibizumabe (tratamento de primeira linha da DMRI), tendo a mesma via de administração e o mesmo mecanismo de ação (anti- VEGF).

O uso off label do Bevacizumabe é novo, datado a partir de 2006, porém os estudos que já foram realizados mostram que a equivalência entre os medicamentos existe e que o tratamento com o Avastin ${ }^{\circledR}$ traz benefícios e melhora para o paciente, além de ter um custo menor. Portanto, conclui-se que o bevacizumabe é o medicamento com o melhor custobenefício para a DMRI.

\section{REFERÊNCIAS}

AMERICAN ACADEMY OF OPHTALMOLOGY. Avastin in Ophthalmology: A Global

Phenomenon. Disponível em: $<$ https://www.aao.org/current-insight/avastin-inophthalmology-global-phenomenon $>$. Acesso em: 31/10/2019.

ANVISA. Câmara de Regulação do Mercado de Medicamentos- CMED Secretaria Executiva PREÇOS MÁXIMOS DE MEDICAMENTOS POR PRINCÍPIO ATIVO AM , AP , BA , CE , MA , MG , PB , PE , PI , ( medicamentos da Portaria MS DEMAIS ESTADOS

Medicamentos Genéricos de SP e MG A SCME. [s.l: s.n.]. v. 914

AVASTIN: Bevacizumab. Responsável Técnico Tatiana Tsiomis Díaz. Rio de Janeiro: Produtos Roche Químicos e Farmacêuticos S.A., 2005. Bula de remédio

BRASIL. MINISTÉRIO DA SAÚDE. ANVISA. Registro de medicamentos. Como a ANVISA vê o uso off label de medicamentos. Disponível em: $<\mathrm{http}$ //www.anvisa.gov.br/medicamentos/registro/registro_offlabel.htm>. Acesso em: 20/04/2019. 
BRASIL. MINISTÉRIO DA SAÚDE. ANVISA. Portaria conjunta nº 18 , de 02 de julho de 2018. Disponível em

$<$ http://portalarquivos2.saude.gov.br/images/pdf/2019/janeiro/08/PCDT-2018-DenegeracaoMacular-1.pdf>. Acesso em: 25/04/2019.

BRATS. Boletim brasileiro de avaliação de tecnologias em saúde. Boletim Brasileiro de Avaliação de Tecnologias em Saúde, p. 1-13, 2008.

CHAKRAVARTHY, U. et al. Ranibizumab versus bevacizumab to treat neovascular agerelated macular degeneration: One-year findings from the IVAN randomized trial.

Ophthalmology, v. 119, n. 7, p. 1399-1411, 2012.

CHEN, Y. H. et al. Evaluation of the sterility, stability, and efficacy of bevacizumab stored in multiple-dose vials for 6 months. Journal of Ocular Pharmacology and Therapeutics, v. 25, n. 1, p. 65-69, 2009.

DAMICO, F. M. Angiogênese e doenças da retina. Arquivos brasileiros de oftalmologia, v. 70, n. 3, p. 547-553, 2007.

EHLERS, J. P. The MANTA 1-year results: The anti-VEGF debate continues. British Journal of Ophthalmology, v. 97, n. 3, p. 248-250, 2013.

EMPTAGE, N. P. et al. American Academy of Ophthalmology Retina/Vitreous Panel. Preferred Practice Pattern ${ }^{\circledR}$ Guidelines. Age-Related Macular Degeneration. American Academy of Ophthalmology, 2015.

FIGUEIREDO, F. D. A.; FIREMAN, M. A. D. A. Protocolo clínico e diretrizes terapêuticas degeneração macular relacionada com a idade (forma neovascular). MINISTÉRIO DA SAÚDE, p. 1-37, 2018.

GASS, J. D. A clinicopathologic study of a peculiar foveomacular dystrophy. Transactions of the American Ophthalmological Society, v. 72, p. 139-156, 1974.

GAZARIAN, M. et al. Off-label use of medicines: Consensus recommendations for evaluating appropriateness. Medical Journal of Australia, v. 185, n. 10, p. 544-548, 2006.

GROUP, C. R. Ranibizumab and Bevacizumab for Neovascular Age-Related Macular Degeneration. New England Journal of Medicine, v. 364, p. 1897-1908, 2011.

KLEIN, R. et al. Fifteen-Year Cumulative Incidence of Age-Related Macular Degeneration. The Beaver Dam Eye Study. Ophthalmology, v. 114, n. 2, p. 253-262, 2007.

KODJIKIAN, L. et al. Ranibizumab versus bevacizumab for neovascular age-related macular degeneration: Results from the GEFAL noninferiority randomized trial. Ophthalmology, v. 120, n. 11, p. 2300-2309, 2013. 
KREBS, I. et al. A randomised double-masked trial comparing the visual outcome after treatment with ranibizumab or bevacizumab in patients with neovascular age-related macular degeneration. British Journal of Ophthalmology, v. 97, n. 3, p. 266-271, 2013.

MARTIN, D. F. et al. Ranibizumab and bevacizumab for treatment of neovascular age-related macular degeneration: Two-year results. Ophthalmology, v. 119, n. 7, p. 1388-1398, 2012.

MICHELS, S. et al. Systemic bevacizumab (Avastin) therapy for neovascular age-related macular degeneration: Twelve-week results of an uncontrolled open-label clinical study. Ophthalmology, v. 112, n. 6, p. 1035- 1047.e9, 2005.

NOVARTIS BIOCIÊNCIAS S.A. Contribuição referente à "Recomendação sobre proposta de incorporação no SUS do medicamento ranibizumabe para tratamento da degeneração macular neovascular (exsudativa ou úmida) relacionada à idade". 2014.

PACKEISER, P. B.; RESTA, D. G. Farmacoeconomia: Uma Ferramenta Para a Gestão Dos Gastos Com Medicamentos Em Hospitais Públicos. Infarma - Ciências Farmacêuticas, v. 26, n. 4, p. 215, 2014.

RADLEY, D. C.; FINKELSTEIN, S. N.; STAFFORD, R. S. Off-label prescribing among office-based physicians. Archives of Internal Medicine, v. 166, n. 9, p. 1021-1026, 2006.

SERRA, D. et al. Análise epidemiológica dos pacientes acometidos por Degeneração Macular Relacionada à Idade, atendidos no Setor de Retina da Faculdade de Medicina do ABC.

Arquivos Brasileiros de Ciências da Saúde, v. 33, n. 1, p. 20-23, 2014.

STEINBROOK, R. The Price of Sight - Ranibizumab, Bevacizumab, and the Treatment of Macular Degeneration. October, p. 1409-1412, 2006.

VAZ CARNEIRO, A.; COSTA, J. Off-label prescription: Practice and problems. Revista Portuguesa de Cardiologia, v. 32, n. 9, p. 681-686, 2013.

ZANINI, A. C. et al. Farmacoeconomia: Conceitos e Aspectos Operacionais. Revista Brasileira de Ciencias Farmaceuticas/Brazilian Journal of Pharmaceutical Sciences, v. 37, n. 3, 2001.

WHO. World Health Organization. Magnitude and causes of visual impairment - No cause for compacency. Disponível em: http://www.icoph.org/downloads/whofactsheet.pdf. Acesso em: 13 de abr 2019.

Publicado em 29/10/2020 